\title{
IMPROVED BOUNDS FOR THE SPREAD OF SPORADIC GROUPS
}

\author{
J. D. BRADLEY AND P. E. HOLMES
}

\begin{abstract}
The spread of a group $G$ is the greatest number $r$ such that, for every set of non-trivial elements $\left\{x_{1}, \ldots, x_{r}\right\}$, there exists an element $y$ with the property that $\left\langle x_{i}, y\right\rangle=G$ for $1 \leqslant i \leqslant r$. In this paper we obtain good upper bounds for the spread of fourteen sporadic simple groups computationally, and we determine the value of the spread of $\mathrm{M}_{11}$ by hand.
\end{abstract}

\section{Introduction}

It was shown by Binder in $[\mathbf{1}, \mathbf{2}]$ that given any two non-trivial elements $x_{1}$ and $x_{2}$ of the symmetric group $\mathrm{S}_{n}, n>4$, there exists a third element $y$ such that $\mathrm{S}_{n}=\left\langle x_{1}, y\right\rangle=\left\langle x_{2}, y\right\rangle$. From this Brenner and Wiegold made the following definition in $[6]$.

Definition 1. Let $r$ be a positive integer. A finite non-abelian group $G$ is said to have spread $r$ if, for every set $\left\{x_{1}, x_{2}, \ldots, x_{r}\right\}$ of distinct non-trivial elements of $G$, there exists an element $y \in G$ such that $G=\left\langle x_{i}, y\right\rangle$ for all $i$, and $r$ is the maximum value for which this is true. We denote the spread of a group $G$ by $s(G)$.

It is clear from the above definition that $s(G)=0$ if $G$ is neither semi-simple (that is, it has no proper soluble normal subgroup) nor the semidirect product of two cyclic groups of prime order, as no element can be one of a generating pair if it is in a normal subgroup with non-cyclic quotient. It is proved in $[11]$ that $s(G) \geqslant 1$ for all finite simple groups $G$.

The generation properties of finite groups, especially finite simple groups, are things that have provoked interest from researchers over the years. An example of such a generation property is the probability of generating a group with a ramdomly chosen element $[\mathbf{1 1}, \mathbf{1 5}]$, but there are many others. The concept of the spread is another such generation property and has been studied, for example, by Guralnick and Shalev using counting and probabilistic methods [12]. The spread of sporadic groups has been studied before by Bradley, Ganief, Moori and Woldar; see below.

Pairwise generating sets are studied in [14] and [3]. These papers are concerned with the maximal size $\mu(G)$ of a set of elements of a group $G$, any two of which generate the whole group. This number is clearly an upper bound for the spread of a group. To see this, let $S$ be a maximal pairwise generating set. Then if there were an element of $G \backslash S$ that generated $G$ with every member of $S$, it would contradict the maximality of $S$. 
It can be observed from Table 1 that the spread of sporadic groups varies wildly, and often bears little relation to the size of the group. For instance, the spread of $\mathrm{M}_{23}$ is much greater than the spread of $\mathrm{M}_{24}$. The groups with a high spread are often the ones with elements of large prime order contained in only a small number of small subgroups. For example, the elements of order 23 in $\mathrm{M}_{23}$ are each contained only in a single subgroup of order $23 \times 11$, and so to obtain a set of elements as described in Section 3 we must have an element from each of these subgroups. In $\mathrm{M}_{24}$, however, the elements of order 23 are contained in one of the 24 subgroups isomorphic to $\mathrm{M}_{23}$, and so it takes far fewer elements to obtain a set of elements of the desired type.

In [10] Ganief and Moori computed lower bounds for the exact spread of the sporadic groups. Breuer et al. used similar methods to improve these bounds in [7]. Upper bounds are given by Moori and the first author in [5].

In this paper we use the computer algebra system MAGMA [4] to calculate much improved upper bounds for the spread of some of the sporadic simple groups, and give a hand proof that $s\left(\mathrm{M}_{11}\right)=3$. The latter result was proved independently by Woldar [19]. His proof differs substantially from ours, and is less geometric. Our results are shown in Table 1. The best known lower bound is given in the second column. The result for $\mathrm{M}_{11}$ comes from our work; the other results come from [7] and [10], which both give a lower bound of 2 for $s\left(\mathrm{M}_{11}\right)$. The third column gives our results. The final column gives the previously known bounds from [5], for comparison purposes.

In Section 2 we give the computer-free proof that the spread of $\mathrm{M}_{11}$ is 3 . Section 3 describes the computational methods used to compute the bounds for the other groups.

We believe that it is essential that we ensure that our computational results are replicable. To this end, we have included the programs in Appendix A, together with the seeds that MAGMA's random processes use to give our exact results.

Table 1: Bounds for the spread of sporadic groups.

\begin{tabular}{lrcc}
\hline Group & Lower bound & Upper bound & Old upper bound \\
\hline $\mathrm{M}_{11}$ & 3 & 3 & 16 \\
$\mathrm{M}_{12}$ & 3 & 9 & 211 \\
$\mathrm{M}_{22}$ & 21 & 26 & 720 \\
$\mathrm{M}_{23}$ & 1525 & 8064 & 41020 \\
$\mathrm{M}_{24}$ & 12 & 56 & 3152 \\
$\mathrm{~J}_{1}$ & 77 & 179 & 5690 \\
$\mathrm{~J}_{2}$ & 5 & 24 & 1071 \\
$\mathrm{~J}_{3}$ & 78 & 597 & 43792 \\
$\mathrm{McL}$ & 71 & 308 & 31184 \\
$\mathrm{HS}$ & 19 & 33 & 1280 \\
$\mathrm{He}$ & 199 & 1223 & 275125 \\
$\mathrm{Co}_{3}$ & 99 & 1839 & 829200 \\
$\mathrm{Suz}$ & 41 & 956 & 532035 \\
$\mathrm{Fi}_{24}^{\prime} 22$ & 14 & 186 & 210897 \\
\hline
\end{tabular}




\section{The exact spread of $\mathrm{M}_{11}$}

\subsection{Background}

The five sporadic simple Mathieu groups, $\mathrm{M}_{11}, \mathrm{M}_{12}, \mathrm{M}_{22}, \mathrm{M}_{23}$ and $\mathrm{M}_{24}$, were discovered in the 19th century by E. Mathieu [16, 17]. The largest of the five groups is $\mathrm{M}_{24}$, which can be defined to be the automorphism group of the unique Steiner system $(5,8,24)$ — that is, a set of 759 subsets of size 8 of a set $\Omega$ of size 24 such that any five of the 24 points lie together in exactly one of the sets of size 8 . We call these sets of size 8 octads. $\mathrm{M}_{24}$ is a 5 -transitive permutation group on 24 points. The binary Golay code is a twelve-dimensional code of length 24 over the field of order 2 spanned by the 759 octads, with addition defined as the symmetric difference of two sets. This code contains: the vectors of weight 0 and 24, 759 vectors of weight 8 (that is, the octads), 759 vectors of weight 16 (that is, the complements of the octads) and 2576 vectors of weight 12 . These sets of size 12 are called dodecads. Sets corresponding to a vector in the Golay code are called $C$-sets. More information about the group $\mathrm{M}_{24}$ can be found in [8] and [9]. In [9, Chapter 12], detailed information can be found about $\mathrm{M}_{24}$ and its subgroup structure, including a way of easily determining whether a given set is a C-set.

The stabiliser of a dodecad (and hence also the complementary dodecad) in $\mathrm{M}_{24}$ is $\mathrm{M}_{12}$. The stabiliser of a dodecad and a point in the dodecad is the group $\mathrm{M}_{11}$. Then $\mathrm{M}_{11}$ is a permutation group on the eleven non-fixed points in one of the dodecads and the twelve points in the complementary dodecad. The point stabiliser in the eleven-point action is $\mathrm{M}_{10} \cong \mathrm{M}_{6} \cdot 2$, and in the twelve-point action the stabiliser is $\mathrm{L}_{2}(11)$.

\subsection{The main result}

THEOREM 1. $s\left(\mathrm{M}_{11}\right)=3$.

Proof. By checking the orders of the maximal subgroups of $\mathrm{M}_{11}$, we see that the only maximal subgroups containing an element of order 11 are the ones isomorphic to $\mathrm{L}_{2}(11)$. Furthermore, by a counting argument, or by observing that each $\mathrm{L}_{2}(11)$ is a stabiliser of a point in the twelve-point action and elements of order 11 act with cycle shape $1^{1} 11^{1}$, we can see that each element of order 11 is contained in precisely one of these subgroups. Therefore, if $y$ is an element of order 11 fixing the point $p$ in the twelve-point action and $x$ is any element not fixing $p$, then $x$ does not lie in a maximal subgroup with $y$ and hence $\langle x, y\rangle=\mathrm{M}_{11}$. In light of this, we seek to prove that for any set of three non-trivial elements $\left\{x_{1}, x_{2}, x_{3}\right\}$ of $\mathrm{M}_{11}$ there is a point $p$ in the twelve-point action not fixed by any of the three elements. Then we choose an element $y$ of order 11 fixing $p$, and since the only maximal subgroup containing $y$ is the stabiliser of $p$ isomorphic to $\mathrm{L}_{2}(11)$, not containing $x_{1}, x_{2}$ or $x_{3}$, we will have $\left\langle x_{1}, y\right\rangle=\left\langle x_{2}, y\right\rangle=\left\langle x_{3}, y\right\rangle=\mathrm{M}_{11}$, and hence $s\left(\mathrm{M}_{11}\right) \geqslant 3$.

Looking at the character table of $\mathrm{M}_{11}$ we see that involutions fix four points in the twelve-point action, and all other non-trivial elements fix fewer than that. This means that if we have a set of three non-trivial elements, unless they are all involutions there must be a point not fixed by any of the three elements. The only possibility is a set of three involutions whose fixed-point sets are pairwise disjoint. Let us assume, without loss of generality, that $D$ is a dodecad containing the point $p$, and that the copy of $\mathrm{M}_{11}$ that we are dealing with is the stabiliser in $\mathrm{M}_{24}$ of $D$ 
and the point $p \in D$. Let $x_{1}, x_{2}, x_{3} \in \mathrm{M}_{11}$ be three involutions, with fixed-point sets $O_{1}, O_{2}$ and $O_{3}$ respectively, such that every point in $\Omega / D$, the complement of $D$, is in one of $O_{1}, O_{2}$ or $O_{3}$. Now $O_{1}, O_{2}$ and $O_{3}$ are octads, so $O_{1}+O_{2}+O_{3}$ is a C-set. By construction, $\mathrm{O}_{1}+\mathrm{O}_{2}+\mathrm{O}_{3}$ contains the dodecad $\Omega / D$, and also the point $p \in D$. The only C-set to strictly contain a dodecad is $\Omega$, and hence $O_{1}+O_{2}+O_{3}=\Omega$. But this is a contradiction, since $|\Omega|=24$ and $\left|O_{i}\right|=8$ and $p \in O_{i}$ for $i \in\{1,2,3\}$. Hence $s\left(\mathrm{M}_{11}\right) \geqslant 3$.

In order to show that $s\left(\mathrm{M}_{11}\right)=3$, we must show that there exists a set of four non-trivial elements $\left\{x_{1}, x_{2}, x_{3}, x_{4}\right\}$ such that one cannot find $y \in \mathrm{M}_{11}$ such that $\left\langle x_{1}, y\right\rangle=\left\langle x_{2}, y\right\rangle=\left\langle x_{3}, y\right\rangle=\left\langle x_{4}, y\right\rangle=\mathrm{M}_{11}$.

We note from the character table that any element of $\mathrm{M}_{11}$ fixes either a point in the eleven-point action or a point in the twelve-point action. Therefore, if we can find a set of four elements of $\mathrm{M}_{11}$ such that every point in $\Omega$ is fixed by one of them, then any element $y$ lies in a maximal subgroup of $\mathrm{M}_{11}$ with at least one of our elements (that is, the point stabiliser of one of the points fixed by $y$ ). In order to prove that such sets of four elements exist, we first prove a lemma.

Lemma 1. Consider the subgroup $\mathrm{M}_{11}$ of $\mathrm{M}_{24}$ fixing the dodecad $D$ and the point $p \in D$. If $O$ is an octad containing $p$ and meeting $D$ in four points, then there is an involution $\pi \in \mathrm{M}_{11}$ fixing $O$ pointwise.

Proof. Consider the fixed-point set of an involution in our copy of $\mathrm{M}_{11}$. It is certainly an octad, it certainly contains $p$ and since it contains three fixed points in the eleven-point action and four in the twelve-point action, it is an octad satisfying the conditions of the lemma. Now let $O$ be an octad satisfying the conditions of the lemma. Since $\mathrm{M}_{11}$ is 4-transitive (and hence 3-transitive) on the eleven points, there is an involution $\pi \in \mathrm{M}_{11}$ fixing the three points of $O$ which lie in $D /\{p\}$. Let $O_{\pi}$ be the fixed point set of $\pi$. The set $O+O_{\pi}$ is a C-set. It is also strictly contained in $\Omega / D$. This means that $O+O_{\pi}$ is the empty set, and hence $O=O_{\pi}$, completing the proof of the lemma.

We now look at a particular copy of $\mathrm{M}_{11}$. Here we use the notation of [9, Chapter $12]$. We take our copy of $\mathrm{M}_{11}$ to be the stabiliser of the following dodecad

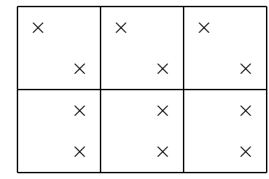

and the point 0 , corresponding to the point in the top left of the array. Consider the following element, well known to be in $\mathrm{M}_{24}$.

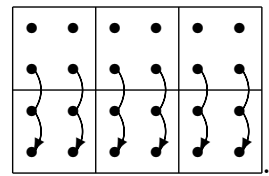

It clearly fixes the point 0 and stabilises the above dodecad, so it is in our copy of $\mathrm{M}_{11}$. In view of Lemma 1, to prove the theorem we need to exhibit three octads all containing 0 , all meeting the dodecad in four points, and such that all 24 points lie 
either in one of our octads or in the fixed-point set of the element of order 3 above. The following three octads will suffice.
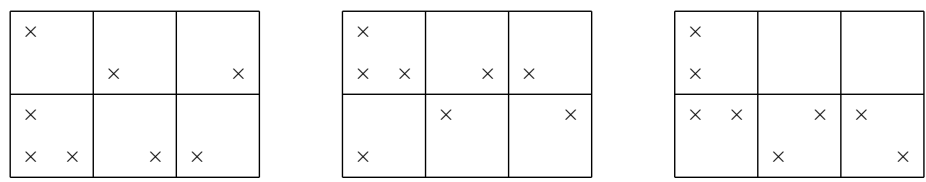

\section{Computational methods}

We use Magma [4] Version 2.18 to obtain upper bounds for the spread of fourteen of the sporadic groups. This section describes the methods used; the programs themselves are in Appendix A.

\subsection{Using coverings}

This section uses the concept of a covering. A set $S$ of proper subgroups of a group $G$ is a covering of $G$ if $G=\bigcup_{s \in S} s$. A minimal covering is a covering of minimal size. The size of a minimal covering is denoted by $\sigma(G)$. We use small and minimal coverings in this section. Minimal coverings of the sporadic groups are studied in [13] and [14].

Table 2 gives $\sigma(G)$ for the sporadic groups studied in this paper. These are all the sporadic groups that have an upper bound for $\sigma(G)$ of less than a million. We provide a single figure when $\sigma(G)$ is known. Otherwise an interval containing $\sigma(G)$ is given.

We use the following lemma.

Table 2: Bounds for the covering numbers of sporadic groups.

\begin{tabular}{lr}
\hline \multicolumn{1}{c}{$G$} & \multicolumn{1}{c}{$\sigma(G)$} \\
\hline $\mathrm{M}_{11}$ & 23 \\
$\mathrm{M}_{12}$ & {$[12,210]$} \\
$\mathrm{M}_{22}$ & 771 \\
$\mathrm{M}_{23}$ & 41709 \\
$\mathrm{M}_{24}$ & 3336 \\
$\mathrm{~J}_{1}$ & {$[5165,5415]$} \\
$\mathrm{J}_{2}$ & {$[907,1154]$} \\
$\mathrm{J}_{3}$ & {$[23648,44100]$} \\
$\mathrm{Fi}_{22}$ & 221521 \\
$\mathrm{Co}_{3}$ & {$[505288,832835]$} \\
$\mathrm{Suz}$ & {$[338625,540333]$} \\
$\mathrm{McL}$ & {$[24541,24553]$} \\
$\mathrm{He}$ & 464373 \\
$\mathrm{HS}$ & 1376 \\
\hline
\end{tabular}


Lemma 2. Let $S$ be a covering of $G$, and $X$ a set of elements of $G$ such that $X \cap s$ is non-empty for each $s \in S$. Then $|X|-1$ is an upper bound for the spread of $G$.

Proof. Suppose that an element $g \in G$ exists with $\langle x, g\rangle=G$ for all $x \in X$. By the definition of a covering, $g$ is contained in some member of $S$, say $s$. The lemma states that $s \cap X$ is non-empty, and so contains some element $x$. So $\langle g, x\rangle \leqslant s<G$.

We consider groups with different types of minimal coverings. Each type of covering requires a different approach, and so do different sizes of group. These approaches are described below.

\subsection{The easy case}

The simplest case is when $G$ is fairly small (in this case $|G| \leqslant\left|\mathrm{M}_{24}\right|$ ), has a minimal covering that is the union of conjugacy classes of low-index maximal subgroups of $G$, and MAgma [4] stores a list of its maximal subgroups. In this case we use the function DoFullClassGroupstrong. The groups successfully treated in this way are $\mathrm{M}_{11}, \mathrm{M}_{22}, \mathrm{M}_{24}$ and $\mathrm{HS}$.

We note that this method gives $s\left(\mathrm{M}_{11}\right) \leqslant 3$, which Section 2 shows is the actual value of $s\left(\mathrm{M}_{11}\right)$. This demonstrates the effectiveness of the program in at least that case.

We use $\mathrm{M}_{22}$ to illustrate the method. By [13] it has a minimal covering consisting of all maximal subgroups isomorphic to $\mathrm{M}_{21}, \mathrm{~L}_{2}(11)$ or $2^{4}: \mathrm{A}_{6}$. This covering has size 771 .

The first task is to compute the permutation representation of $G$ on the 771 cosets of subgroups in the covering. This is done by the function MakeGroup, which also returns the images in this representation of a representative of each conjugacy class of elements.

Next, the function MakeElts computes a set $F$ consisting of all non-trivial maximal fixed-point sets in this representation. By this we mean all maximal subsets of points $s$ such that there exists a non-trivial element of $G$ that fixes $s$ pointwise, and $s$ is not the full set. This is all we need, by Lemma 2.

Finally, StrongSearch searches for a small set $X \subset F$, where $\bigcup_{x \in X} x$ is the whole set of points. With each pass through the loop, it collects from $F$ all optimal candidates for the next member of $X$, and adjoins a random one of these to $X$.

\subsection{Variations on the theme}

When the group is larger, then there are likely to be a large number of maximal fixed-point sets. In this case, we cannot store them all, so we use one of the functions DoXXXClassGroupFast. These two functions call FastSearch instead of StrongSearch. This function does not search the whole of $F$ for optimal candidates each time. Instead, it takes a small sample $F^{\prime}$ from $F$, and then adjoins an optimal choice from $F^{\prime}$ to $X$.

Additional savings in space and time are given by not explicitly computing the whole of $F$. The function MakeEltsWeak computes at least one maximal fixed-point set from each $G$-orbit, and possibly some which are not maximal. The responsibility for making other members of the $G$-orbits is passed to FastSearch, which makes only those that it needs for creating the sets $F^{\prime}$.

We use DoFullClassGroupFast for the groups $\mathrm{M}_{23}$ and $\mathrm{J}_{3}$. 
Sometimes, $G$ has a minimal covering $C$ that is not a union of conjugacy classes of maximal subgroups. This case is dealt with using the functions DoPartialClassGroupXXX. This begins by assuming that the covering is in fact $\bar{C}$, the smallest covering that contains $C$ as a subset but is closed under conjugacy in $G$. It then calls MakeNeeds to create a subset $P$ of the points, where the set of the point stabilisers in $P$ is a covering of $G$. It passes this set to one of the two Search functions, where it is used instead of the full point set. All members of $F$ are replaced by their intersections with $P$.

The groups that needed DoPartialClassGroupStrong were $\mathrm{M}_{12}, \mathrm{~J}_{1}$ and $\mathrm{J}_{2}$. For McL, we used DoPartialClassGroupFast.

When a minimal covering $C$ for $G$ is too large for the above methods, then we can use the orbits of a subgroup. (At the time of writing, 'too large' means 'over 100000 subgroups'.) We look at the orbits of subgroups in their action on $P$. We choose a subgroup $K$ that has $n$ orbits on $P$ for some $n<100000$, and where most of these orbits are regular. The functions XXXWithOrbits use the above methods to find $P^{\prime} \subset P$ such that $P^{\prime}$ intersects every $K$-orbit non-trivially and $P^{\prime}=\bigcup X$ for a small set $X \subset F$. Then it is clear that

$$
P=\bigcup_{x \in X} x^{K}
$$

so $|K| .|X|-1$ is an upper bound for the spread of $G$.

The function DoFullClassGroupWithOrbits dealt with the groups $\mathrm{Fi}_{22}, \mathrm{Co}_{3}$, Suz and He. The subgroup $K$ is a cyclic group of order 11, 23, 11 and 17 respectively.

\subsection{Obtaining the larger groups}

If readers wish to verify our calculations, then it is important that they use identical input. Not all the permutation groups that we use are available with their maximal subgroups in MAGMA, so this section gives the necessary details for the reader to recreate these representations. The groups concerned are $\mathrm{Fi}_{22}$ and Suz.

All representations are taken from the Web Atlas [18]. The input to MAgma was an eight-generator group for Suz and a ten-generator group for $\mathrm{Fi}_{22}$. In both cases, the first two generators were the group generators in the smallest permutation representation as given in the Web Atlas. The other generators come in pairs, and each pair generates a maximal subgroup. These were obtained by using the words for maximal subgroups given in the Web Atlas.

The extra generators for Suz generate $2^{1+6} \cdot \mathrm{U}_{4}(2), \mathrm{U}_{5}(2)$ and $\mathrm{J}_{2}: 2$, in that order. The subgroups of $\mathrm{Fi}_{22}$ given by the extra generators are $\mathrm{U}_{6}(2), \mathrm{O}_{7}(3), \mathrm{O}_{8}^{+}(2) \cdot \mathrm{S}_{3}$ and $2^{10}: \mathrm{M}_{22}$.

\section{Conclusions}

We have given a hand proof that $s\left(\mathrm{M}_{11}\right)=3$. Computational methods have given us good upper bounds for $s(G)$ for thirteen of the other sporadic groups.

This deals with all sporadic groups known to have a covering of size less than a million. The two groups that seem most open to attack next are $\mathrm{CO}_{2}$ and $\mathrm{Ru}$, as these have minimal coverings of sizes at most 5 and 12 million respectively.

We note that better results were obtained for some of the groups in trial runs, but our table gives only the results that were given by known seeds. 
Acknowledgements. The second author is supported by by a Royal Society Dorothy Hodgkin fellowship and the EPSRC grant EP/C523229/1 held by members of the CIRCA group in St Andrews. We would like to thank Robert Curtis for a discussion about Section 2. We would also like to thank Jamshid Moori for useful discussions on the subject of the spread of sporadic simple groups.

\section{Appendix A. Links to MATHEMATICA notebooks}

The programs used to generate the results presented in the paper, together with the seeds used by MAGMA's random processes to give the exact results, can be found at:

$$
\text { http://www.Ims.ac.uk/jcm/10/lms2006-022/appendix-a. }
$$

\section{References}

1. G. Binder, 'The bases of the symmetric group', Izv. Vyss. Ucebn. Zaved. Mathematika 78 (1968) 19-25. 132

2. G. Binder, 'The two element bases of the symmetric group', Izv. Vyss. Ucebn. Zaved. Mathematika 90 (1970) 9-11. 132

3. S. BlaCKBURn, 'Sets of permutations that generate the symmetric group pairwise', J. Combin. Theory Ser. A 113 (2006) 1132-1138. 132

4. W. Bosma and J. J. Cannon, Handbook of Magma functions (School of Mathematics and Statistics, University of Sydney, Sydney, 1995). 133, 136, 137

5. J. D. Bradley and J. Moori, 'On the exact spread of sporadic simple groups', Comm. Algebra, to appear. 133

6. J. L. Brenner and J. Wiegold, 'Two generator groups, I', Michigan Math. J. 22 (1975) 53-64. 132

7. T. Breuer, R. M. Guralnick and W, M. Kantor, 'Probabilistic generation of finite simple groups II', J. Algebra, to appear. 133

8. J. H. Conway, R. T. Curtis, S. P. Norton, R. A. Parker and R. A. Wilson, Atlas of finite groups (Clarendon Press, 1985). 134

9. J. H. Conway and N. A. Sloane, Sphere packings lattices and groups. (Springer, 1988). 134, 135

10. S. Ganief and J. Moori, 'On the spread of sporadic simple groups', Comm. Algebra 29 (2001) 3239-3255. 133

11. R. M. Guralnick and W. M. Kantor, 'Probabilistic generation of finite simple groups', J. Algebra 234 (2000) 743-792. 132

12. R. M. Guralnick and A. Shalev, 'On the spread of finite simple groups', Combinatorica 23 (2003) 73-87. 132

13. P. E. Holmes, 'Subgroup coverings of some sporadic groups', J. Combin. Theory, Ser. A, to appear. 136, 137

14. P. E. Holmes and A. MARóti, 'Covering and generating sporadic simple group pairwise', Preprint, CIRCA, St. Andrews, 7 (2006). 132, 136 
15. W. M. KANTOR and A. LuBotzky, 'The probability of generating a finite classical group', Geom. Dedicata 36 (1990) 67-87. 132

16. E. Mathieu, 'Memoire sur l'etude des fonctions de plusieurs quantites', J. Math. Pures Appl. 6 (1861) 241-243. 134

17. E. Mathieu, 'Sur les fonctions cinq fois transitives de 24 quantites', J. Math. Pures Appl. 18 (1873) 25-46. 134

18. R. A. Wilson, et al., 'A World-Wide-Web Atlas of group representations', http://brauer.maths.qmul.ac.uk/Atlas/v3/. 138

19. A. WoldAR, 'The exact spread of the Mathieu group $\mathrm{M}_{11}$ ', J. Group Theory, to appear. 133

J. D. Bradley johnbradley120@gmail.com

National University of Rwanda

Butare

Rwanda

P. E. Holmes peh27@dpmms.cam.ac.uk

DPMMS

Centre for Mathematical Sciences

Cambridge CB3 0BW

United Kingdom 